\title{
Una mirada al fenómeno de la contabilidad creativa desde las NIIF y las NICSP
}

\section{REINIER HOLLANDER SANHUEZA ${ }^{a}$, FERNANDO MORALES PARADA ${ }^{b}$}

\section{pp. $\mathbf{8 5 - 9 4}$}

\begin{abstract}
RESUMEN El presente artículo cumple con la finalidad de identificar y describir las principales posibilidades de aplicación de la contabilidad creativa en el marco de las normas internacionales de información financiera y de las normas internacionales de contabilidad del sector público, demostrando la existencia de opcionalidades y subjetividades de las normas internacionales, para con esto determinar las posibles prácticas de contabilidad creativa. Con el fin de lograr lo mencionado, se desarrolla un análisis en el que se presentan algunos ejemplos de las normas, identificando en estas las opcionalidades -es decir, cuando se presentan, ante un hecho económico, distintas alternativas de contabilización-, y las subjetividades, o sea, situaciones en las que un juicio profesional cobra una gran relevancia debido a que es sujeto de interpretación. Del estudio se concluye que las normas permiten una variedad de posibilidades, en especial aquellas relacionadas con la regulación de partidas de activos y en donde las técnicas manipuladoras están mayormente centradas en la opcionalidad de la aplicación de la norma por sobre la subjetividad.
\end{abstract}

PALABRAS CLAVE contabilidad, contabilidad creativa, IFRS, IPSAS, NICSP, NIIF.

\section{HISTORIA DEL ARTÍCULO}

¿CÓMO CITAR?:

Hollander-Sanhueza, R. \& MoralesParada, F. (2017). Una mirada al fenómeno de la contabilidad creativa desde las NIIF y las NICSP. Perspectiva Empresarial, 4(1), 85-94. http://dx.doi.org/10.16967/rpe. v4n1a6

RECIBIDO: 5 de diciembre de 2016 APROBADO: 3 de febrero de 2017

CORRESPONDENCIA:

Reinier Hollander Sanhueza Av. Collao 1202 Facultad de Ciencias Empresariales, Universidad del BioBio, Concepción, Región del Bio-Bio, Chile.

a Académico del Departamento de Administración y Auditoría, Facultad de Ciencias Empresariales, Universidad del Bío-Bío, Concepción-Chile. Correo electrónico: rholland@ubiobio.cl

b Director de Finanzas y Administración, académico del Departamento de Administración y Auditoría, Facultad de Ciencias Empresariales, Universidad del Bío-Bío, Concepción-Chile. Correo electrónico: fmorales@ubiobio.cl 


\section{¿CÓMO CITO EL ARTíCULO?} HOW TO CITE THIS PAPER?

\section{CHICAGO:}

Hollander-Sanhueza, Reinier y Morales-Parada, Fernando. 2017. "Una mirada al fenómeno de la contabilidad creativa desde las NIIF y las NICSP". Perspectiva Empresarial 4(1): 85-94. http://dx.doi. org/10.16967/rpe.v4n1a6

MLA:

Hollander-Sanhueza, Reinier y Morales-Parada. “Una mirada al fenómeno de la contabilidad creativa desde las NIIF y las NICSP". Perspectiva Empresarial 4.1 (2017): 8594. Digital. http://dx.doi. org/10.16967/rpe.v4n1a6

\section{A Perspective on the Creative Accounting Phenomenon form IFRS and IPSAS}

ABSTRACT This paper identifies and describes the main possibilities to apply creative accounting within the framework of the international financial information standards and the international public sector accounting standards, showing the existence of loopholes and subjectivity in international standards, in order to determine possible creative accounting practices. For such purpose, an analysis is made and some examples of the standards are given, by identifying their loopholes - i.e., when they arise before an economic event and/or several accounting alternatives-, and subjectivities in which the professional judgement becomes significantly relevant since it is an interpretation subject. Results show that the standards allow several possibilities, especially those related to the regulation of asset items and in those in which manipulative techniques are mainly focused on the applicability options of standards over subjectivity.

KEYWORDS accounting, creative accounting, IFRS, IPSAS.

\section{Um olhar ao fenômeno da contabilidade criativa da perspectiva das NIIF e das NICSP}

RESUMO 0 presente artigo cumpre com a finalidade de identificar e descrever as principais possibilidades de aplicação da contabilidade criativa sob a perspectiva das normas internacionais de informação financeira e das normas internacionais de contabilidade do setor público, mostrando a existência de alternativas e subjetividades às normas internacionais para determinar as práticas possíveis de contabilidade criativa. Com o fim de conseguir o mencionado, desenvolve-se uma analise na que se apresentam alguns exemplos das normas, identificando estas alternativas - Ou seja, quando se apresentam, diante um fato econômico, diferentes alternativas de contabilização-, e as subjetividades, ou situações nas que um juízo profissional se torna relevante devido a que é sujeito de interpretação. Do estudo conclue-se que as normas permitem uma variedade de posibilidades, principalmente aquelas relacionadas com a regulamentação das contas de ativos e onde as técnicas manipuladoras estão maiormente focadas nas alternativas da aplicação da norma por cima da subjetividade.

PALAVRAS CHAVE contabilidade, contabilidade criativa, IFRS, IPSAS, NICSP, NIIF. 


\section{Introducción}

La información contable como parte de las decisiones empresariales constituye un elemento clave. Es reconocida la importancia de la contabilidad en el quehacer empresarial, y lo confirma su trayectoria. A pesar de ello, la contabilidad como instrumento de medición presenta falencias, que se han acentuado por los fuertes cambios empresariales, como la internacionalización de las empresas, la figura de los stakeholders como usuarios de la información financiera y la velocidad de operación de los mercados financieros, entre otros.

Algunas de sus debilidades es la falta de uniformidad en la valoración de las partidas, las normas no cubren todas las situaciones que deben ser registradas, o a pesar de que exista regulación, muchas veces son las mismas normas las que permiten alternativas en la valorización contable, o a veces su redacción no es suficientemente clara, lo que posibilita a los contables la libre interpretación, provocando con esto que ante iguales hechos económicos se produzcan diferentes contabilizaciones. Esta realidad existente en las normas, reconocida como contabilidad creativa, muchas veces es aprovechada por la gerencia o la administración de las empresas, al utilizar la contabilidad para encauzar los resultados, o bien algunas partidas, hacia lo que ellos desean obtener a una fecha determinada, por sobre el cauce natural que resultaría de no manipular las cifras contables.

\section{El concepto de contabilidad creativa}

La contabilidad creativa utiliza las posibilidades asociadas con opcionalidad, subjetividad y vacíos de la norma para presentar estados financieros que proyecten una imagen deseada y no necesariamente la que en realidad es. Amat \& Blake (1999) reconocen este efecto como un proceso mediante el cual los contables sacan partido de su conocimiento de las normas para manipular las cifras incluidas en las cuentas de una empresa.

En la definición de Naser (1993), este la señala como el proceso de manipular la contabilidad aprovechándose de las reglas y las opciones de valuación y revelación práctica para transformar los estados financieros, de lo que son a lo que deben ser. Complementa indicando que la flexibilidad en las valuaciones de contables y normas de revelación siempre ha permitido algún grado de creatividad. Por su parte, Jameson (1988) afirma que es esencialmente un proceso de usar las reglas, la flexibilidad proporcionada y las omisiones de las normas de contabilidad.

Los alcances de la contabilidad creativa pueden tener efecto en cualquier tipo de partida de los estados financieros, es decir, tanto en balance como en resultado (pérdidas y ganancias), así como podrá afectar la valoración o la revelación. Por otra parte, la alteración contable puede implicar el mejoramiento o empeoramiento financiero (en cualquiera de las partidas) según sea el objetivo deseado por parte del manipulador, cuestión que se profundiza en el apartado siguiente. Es interesante resaltar que la aplicabilidad de la contabilidad creativa se basa fundamentalmente en la aplicabilidad creativa de las normas y no en otras formas de manipulaciones para lograr estados financieros deseados por sobre lo que las normas en su "normal" aplicación resultarían.

\section{Causa y finalidad de la contabilidad creativa}

Naser (1993, p. 59) enumera una serie de causas que estarían provocando un incremento de las prácticas de contabilidad creativa. Entre otras, enuncia la desinformación, los problemas de agencia y costos políticos, la incertidumbre y las presiones de inversionistas institucionales. Pero quizás un punto determinante que no menciona este autor, y que es causa fundamental de la existencia de la contabilidad creativa, es la característica de las normas, que son el medio por el cual se facilitaría o dificultaría la aplicabilidad de estas prácticas. Al respecto, Laínez \& Callao (1999, p. 27) profundizan en este aspecto ampliando sobre la discrecionalidad en la aplicación de determinados principios contables, prevalencia de la imagen fiel como referencia en la elaboración de la información, necesidad de efectuar estimaciones y subjetividad en la aplicación de determinados criterios contables, flexibilidad en las normas al contemplar distintas opciones para reflejar un mismo hecho u operación y la existencia de vacíos en la regulación.

Pero ese fin de alterar artificialmente los estados financieros sobre su aplicación natural de la norma persigue diversos objetivos. Es así como Healy \& Wahlen (1999) logran ver tres objetivos 
principales: los procedentes de la valoración de acciones, los relativos a acuerdos y contratos relacionados con cifras contables y los objetivos de carácter político y social. Por otra parte, y con algunas coincidencias, Amat \& Blake (1999, p. 18) plantean que tras la manipulación de cuentas, los directivos de empresas que cotizan persiguen la estabilización de ingresos (prefieren reflejar estabilidad en el crecimiento del beneficio), ayudar a mantener o inflar el precio de las acciones, y si los directivos están comprometidos en operaciones internas con las acciones de su empresa, pueden utilizar la contabilidad creativa para retrasar la llegada de información al mercado para beneficiarse de su información privilegiada (insider trading).

La investigación desarrollada por Roychowdhury (2006) establece que los gerentes manipulan actividades reales para evitar reportar informes anuales de pérdidas. En concreto, presenta evidencia que sugiere descuentos de precios para aumentar el volumen de ventas; también las empresas analizadas realizan un incremento de la producción para presentar menores costos de los bienes vendidos y reducción de los gastos para mejorar los márgenes reportados.

Gracia \& Lopera (2014) plantean diferentes posiciones en torno a las causas e impactos de la contabilidad creativa, que van desde la válida aplicación del juicio profesional hasta la manipulación fraudulenta de las cifras contables.

Interesante es el artículo de Black, Christensen, Taylor \& Schmardebeck (2016), que se enfoca a la gestión de los ingresos reales como un método cada vez más común de la manipulación de los resultados financieros. En la misma dirección, un estudio realizado por Cheng \& Warfield (2005) demuestra comportamiento oportunista de la gerencia relacionado con los planes de compensación. Si bien la compensación basada en las acciones y la propiedad puede dar resultados positivos, por efectos de incentivo, también puede tener efectos secundarios, como el aumento de la gestión "creativa" de los ingresos.

\section{Objetivo y método}

Este ensayo se ha propuesto identificar, describir y explicar algunas de las posibilidades de aplicación de contabilidad creativa que se podrían llevar a cabo en el marco de la convergencia a las normas internacionales de contabilidad, tanto en NIIF y NICSP, de tal forma que contribuya al conocimiento en la materia a los profesionales contables que se desenvuelven en ambos ámbitos de acción, privado y público.

Del planteamiento del objetivo general es posible especificar el siguiente objetivo en particular: determinar las posibles prácticas de aplicación de contabilidad creativa al momento de aplicar las normas de información financiera NIIF en su versión Full castellano emitida por IASB y en las NICSP. Este objetivo se sitúa en tener observancia de las posibilidades de opcionalidades y subjetividades que ofrece la normativa contable internacional, e incluso los vacíos que pudieran hoy afectar la toma de decisiones de gestión contable.

La metodología consistió en la revisión de algunas normas, que se presentarán como ejemplos, buscando las opciones que existían de contabilizar de una u otra forma, así como conceptos que sugirieran subjetividad por parte del contador, al reconocer, valorizar o revelar información financiera. Este trabajo no pretendió hacer una revisión exhaustiva de cada norma, ni mucho menos comparar NIIF con NICSP, sino enseñar al lector de forma ejemplificadora cómo se reconoce el fenómeno de contabilidad creativa en las normas.

\section{Desarrollo}

\section{Posibilidades de contabilidad creativa en la normativa}

Respecto a las posibilidades de prácticas contables creativas, se podría afirmar que no tienen límite, tal como el propio significado de la palabra "creativo" o "creatividad" invita a pensar en la originalidad. No existe un recetario único sobre las prácticas creativas, aun cuando algún autor haya publicado alguna vez un vademecum de la contabilidad creativa. Diversos textos se han dedicado a describir las posibilidades más comunes de contabilidad creativa (Naser, 1993; Jameson, 1988) o particularidades de normas locales de países específicos (Laínez \& Callao, 1999; Amat \& Blake, 1999; Huber, 1999).

A propósito del advenimiento de la aplicación de las Normas Internacionales de Información 
TABLA 1. Definiciones y reflexiones acerca de contabilidad creativa por diversos autores

\begin{tabular}{|c|c|c|c|}
\hline AUTOR & AÑO & DEFINICIÓN O REFLEXIÓN & REFERENCIA \\
\hline Griffiths & 1988 & $\begin{array}{l}\text { "Todas las empresas de este país [por Gran Gretaña] manipulan sus } \\
\text { beneficios. Todas las cuentas que se publican se basan en libros que han } \\
\text { sido retocados con más o menos delicadeza; las cifras que se facilitan } \\
\text { al público inversor han sido modificadas para proteger al culpable. Es la } \\
\text { estratagema de mayor envergadura desde el caballo de Troya". }\end{array}$ & $\begin{array}{l}\text { Cano (2001), } \\
\text { Huber (1999), Laínez } \\
\text { \& Callao (1999), } \\
\text { Amat \& Blake (1999) }\end{array}$ \\
\hline Smith & 1992 & $\begin{array}{l}\text { "Nos da la impresión que el gran aparente crecimiento ocurrido en los } \\
\text { ochenta, ha sido más resultado del juego de maniobras contables que del } \\
\text { genuino crecimiento económico". }\end{array}$ & Amat \& Blake (1999) \\
\hline $\begin{array}{l}\text { Pasqualini } \\
\& \text { Castel }\end{array}$ & 1993 & $\begin{array}{l}\text { "Trata de poner los medios para dar respuesta a la imaginación de los } \\
\text { nuevos productos financieros". }\end{array}$ & Rodríguez (1996) \\
\hline Pijper & 1993 & $\begin{array}{l}\text { "La contabilidad podría ser usada como una herramienta para distorsionar } \\
\text { la realidad subyacente a la vista de los usuarios de los estados } \\
\text { financieros". }\end{array}$ & $\begin{array}{l}\text { Huber (1999), } \\
\text { Rodríguez (1996) }\end{array}$ \\
\hline Stolowy & 1994 & $\begin{array}{l}\text { "De manera simplificada, la contabilidad llamada creativa apunta a dos } \\
\text { objetivos: mejorar las cuentas y traducir la creatividad financiera". }\end{array}$ & Rodríguez (1996) \\
\hline $\begin{array}{l}\text { Healy \& } \\
\text { Wahlen }\end{array}$ & 1998 & $\begin{array}{l}\text { "La manipulación de las cifras contables tiene lugar cuando los directivos } \\
\text { usan su propio juicio en la elaboración de la información financiera y en } \\
\text { la estructura de transacciones, con la finalidad de alterar los informes } \\
\text { contables para confundir a los usuarios de dicha información acerca de la } \\
\text { realidad económica de la empresa”. }\end{array}$ & Cano (2001) \\
\hline $\begin{array}{l}\text { Degeorge } \\
\text { et al. }\end{array}$ & 1999 & $\begin{array}{l}\text { "Consiste en el ejercicio estratégico de la discreción de los directivos para } \\
\text { influir sobre el resultado publicado". }\end{array}$ & Vidal (2002) \\
\hline $\begin{array}{l}\text { Dechow } \\
\text { et al. }\end{array}$ & 1999 & $\begin{array}{l}\text { "Se trata de prácticas favorecidas por la flexibilidad de la normativa } \\
\text { contable y encaminadas a la consecución de una determinada posición } \\
\text { previamente establecida por la dirección, que dista en numerosas } \\
\text { ocasiones de la verdadera situación económico-patrimonial de la } \\
\text { empresa". }\end{array}$ & Vidal (2002) \\
\hline
\end{tabular}

Fuente: elaboración propia a partir de los autores señalados

Financiera (NIIF) en Europa y en diversos países alrededor del mundo, se ha manifestado la preocupación por estudiar en las normas internacionales las posibilidades que ofrecen estas respecto a prácticas manipuladoras. Es decir, el problema de subjetividades, opcionalidades y vacíos igualmente estaría presente en las NIIF (Amat \& Perramon, 2005; García \& Zorio, 2004).

Sin embargo, estas posibilidades locales o internacionales rondan en similares aspectos de la contabilidad, muchos de ellos coincidentes, como son la utilización por provisiones o revalorizaciones en determinadas cuentas, o aspectos más específicos como operaciones de leasing (arrendamientos), instrumentos financieros o tratamiento de los intangibles. Al respecto, Stolowy \& Breton (2004), como parte de la revisión bibliográfica de su estudio, identifican a diversos autores que han examinado determinadas posibilidades creativas dentro de la contabilidad.

\section{Advenimiento de la aplicación de las normas internacionales de contabilidad en Chile}

Con la entrada en vigencia de la aplicación de las normas internacionales de contabilidad en Chile, y en razón de la convergencia actual de las normas internacionales para el sector público, es necesario analizar los efectos potenciales de prácticas relacionadas con la contabilidad creativa, es decir, las subjetividades y opcionalidades de las normas.

Una visión internacional la proporcionan García \& Zorio (2004), quienes ven en las NIIF los mismos problemas de posibilidades creativas con contabilidades locales de todo el mundo. A modo de ejemplo: en el activo fijo merecen una atención especial las posibilidades en materia de intercambio de activos, revalorización, amortización, saneamiento y capitalización de intereses. Respecto 
a activos intangibles y financieros, destacan las oportunidades que brindan las normas internacionales de ejercer prácticas contables creativas en el ámbito de los bienes intangibles (principalmente gastos de investigación y desarrollo), el inmovilizado financiero y los gastos amortizables. También observan estas posibilidades en provisiones y contingencias, instrumentos financieros y tratamiento de moneda extranjera.

Es interesante destacar que Chile vivió una convergencia que lo llevó en un mediano plazo a adoptar las NIIF, dejando atrás su regulación local (basada en principios y boletines técnicos) y no considerando la adaptación a NIF-Chile ${ }^{1}$, desde el año 2009 en adelante, de forma gradual. Sin embargo, en ningún caso evitaría las manipulaciones contables, pues como ya lo han evidenciado García \& Zorio (2004) y Amat \& Perramon (2005), las NIIF sufren de los mismos problemas de opcionalidad y subjetividad, y actualmente aun habría vacíos en lo que respecta a la valuación y revelación de determinadas partidas contables. Además, Chile actualmente vive la convergencia del sector público, que va desde 2016 hasta 2019, de forma gradual, con excepciones de diversos tipos en su andar por la complejidad, que significa que toda la administración del Estado adopte normas internacionales y con base en la adaptación a las NICSP de la Federación Internacional de Contadores (IFAC por sus siglas en inglés), en el caso chileno, bajo la instrucción y norma emitida por la Contraloría General de la República, Resolución 16 CGR. La incorporación del sector público en estas normas se justifica por las siguientes variables que afectan esta transición²:

a. Transparencia del sistema económico mundial.

b. Fuerte impulso de organismos internacionales.

c. Confiabilidad de las cifras de gobiernos y entidades públicas.

1 Corresponden a las normas de información financiera, las cuales fueron adaptaciones de las NIIF realizadas por el Colegio de Contadores de Chile A.G.

2 Presentación del señor contralor general sobre el proceso de convergencia a NICSP en el Foro de Tesorerías Gubernamentales de América Latina, febrero de 2014. d. Convergencia al estándar NIIF en las empresas chilenas.

e. Rendición financiera para una mayor confianza pública.

f. El ciudadano como (exigente) "accionista" del ente público.

\section{Análisis de las posibilidades de contabilidad creativa en la norma}

El presente apartado detalla las principales posibles prácticas manipuladoras que la norma chilena permitiría, respecto a subjetividades y opcionalidades que se pueden identificar, revisando detallada y cuidadosamente cada proyecto de convergencia (NIF-Chile). Lo anterior, con base en trabajos previos (Morales \& Hollander, 2007 y 2009).

La tabla 2 que aparece a continuación revela de forma resumida la situación respecto a la contabilidad creativa que sería permisible en la norma chilena de general aceptación.

Como se observa en la tabla, hay un total de 63 posibilidades de prácticas creativas en las normas internacionales de contabilidad, versión otrora convergencia chilena (pero cabe recordar que finalmente se optó por NIIF). Detallando la información y separando por posibilidades de subjetividades y opcionalidades que presenta la norma, se obtiene la siguiente tabla 3.

Con base en lo anterior (tabla 3), las mayores posibilidades de prácticas creativas se dan en las opcionalidades, con 51\%. Este resultado atenta contra la comparabilidad de la información financiera, puesto que un hecho económico, dependiendo de las subjetividades y opcionalidades de la norma, puede ser reflejado de distintas maneras y con distintos montos. La comparabilidad de la información financiera se ha utilizado como fortaleza de la existencia de un único modelo contable a nivel internacional, pero como se puede analizar en este trabajo, con la existencia de 63 posibilidades de contabilidad creativa, se amplía el campo de acción del agente creativo que puede utilizar esta debilidad de la norma para presentar una posición financiera que esté de acuerdo con sus intereses. 
TABLA 2. Resumen de las posibilidades creativas descubiertas en la norma

\begin{tabular}{|c|c|c|}
\hline PARTIDA QUE IMPACTA & NORMATIVA ASOCIADA & $\begin{array}{l}\text { N. }{ }^{\circ} \text { DE POSIBILIDADES } \\
\text { CREATIVAS }\end{array}$ \\
\hline Instrumentos financieros & $\mathrm{NIC} n .^{\circ} 39$ & 2 \\
\hline Activos fijos & $\mathrm{NIC} n .^{\circ} 16$ & 5 \\
\hline Activos en arriendo & NIC n. ${ }^{\circ} 17, \mathrm{SIC}^{\star} 15, \mathrm{SIC} 27$ & 1 \\
\hline Transacciones en moneda extranjera & $\mathrm{NIC} \mathrm{n.}{ }^{\circ} 21$ & 5 \\
\hline Gastos financieros & $\mathrm{NICn} \cdot{ }^{\circ} 23$ & 2 \\
\hline Inversiones inmobiliarias & $\mathrm{NIC} n .^{\circ} 40$ & 2 \\
\hline Activos intangibles & NIC n. ${ }^{\circ} 38$ & 3 \\
\hline Deterioro de valor de los activos & NIC n. ${ }^{\circ} 36$ & 5 \\
\hline Combinación de negocios & NIIF n. ${ }^{\circ} 3$ & 2 \\
\hline Inversión en empresas coligadas & NIC n. ${ }^{\circ} 28$ & 1 \\
\hline Participación en negocios conjuntos & $\mathrm{NIC} n .^{\circ} 31$ & 4 \\
\hline Existencia & $\mathrm{NIC} .^{\circ} 2$ & 3 \\
\hline Subvenciones gubernamentales & $\mathrm{NIC}$ n. ${ }^{\circ} 20$ y SIC* 10 & 5 \\
\hline Contratos de construcción & $\mathrm{NIC} n .^{\circ} 11$ & 3 \\
\hline Agricultura & $\mathrm{NIC} n .^{\circ} 41$ & 1 \\
\hline Beneficios a los empleados & NIC n. ${ }^{\circ} 19$ & 3 \\
\hline Planes y beneficios por retiro & $\mathrm{NICn} \cdot{ }^{\circ} 26$ & 1 \\
\hline Utilidad por acción & $\mathrm{NIC}$ n. ${ }^{\circ} 33$ & 1 \\
\hline Ingresos ordinarios & $\mathrm{NIC}$ n. ${ }^{\circ} 18, \mathrm{SIC}^{\star} 27, \mathrm{SIC}^{\star} 31$ & 2 \\
\hline Información financiera intermedia & $\mathrm{NIC}$ n. ${ }^{\circ} 34$ & 1 \\
\hline Hechos posteriores a la fecha del balance & $\mathrm{NIC} n .^{\circ} 10$ & 2 \\
\hline Estados financieros consolidados & $\mathrm{NIC} n .^{\circ} 27$ & 2 \\
\hline Información financiera por segmentos & $\mathrm{NIC} n .^{\circ} 14$ & 2 \\
\hline Políticas contables, cambios en estimaciones y errores & NIC n. ${ }^{\circ} 8$ & 3 \\
\hline Estado de flujo de efectivo & $\mathrm{NIC} n .^{\circ} 7$ & 1 \\
\hline Información financiera en economías hiperinflacionarias & NIC n. ${ }^{\circ} 29$ & 1 \\
\hline Total & & 63 \\
\hline
\end{tabular}

Fuente: Morales \& Hollander, 2007

*Standard Interpretations Committee, (Comité de interpretación de Normas Internacionales de Contabilidad).

TABLA 3. Resumen de las posibilidades creativas observadas en la norma

\begin{tabular}{lcc}
\hline TIPO DE POSIBILIDAD CREATIVA & N. ${ }^{\circ}$ DE POSIBILIDADES & $\%$ \\
\hline Subjetividad & 23 & $37 \%$ \\
\hline Opcionalidad & 32 & $51 \%$ \\
\hline Subjetividad y opcionalidad & 8 & $12 \%$ \\
\hline Total & 63 & $100 \%$ \\
\hline
\end{tabular}

Fuente: elaboración propia 
Ejemplos sobre comparación sobre posibilidades de contabilidad creativa entre NIIF y NICSP en algunas partidas

A continuación se presenta un cuadro resumen con las posibilidades de contabilidad creativa, en el que se hace una comparación entre las NICSP (versión IFAC) y las NIIF (versión IASB). Esta comparación se expone como ejemplo, y no pretende considerar todas las posibilidades de contabilidad creativa existentes.

CUADRO 1. Posibilidades de contabilidad creativa en las existencias.

\begin{tabular}{lll}
\hline & NICSP $^{\circ}{ }^{\circ} \mathbf{1 2}$ & NIC n. ${ }^{\circ} \mathbf{2}$ \\
\hline $\begin{array}{l}\text { SUBJETIVIDAD } \\
\text { VALORATIVA }\end{array}$ & $\begin{array}{l}\text { Las técnicas para la medición del costo de los } \\
\text { inventarios, tales como el método del costo } \\
\text { estándar, pueden ser usadas por conveniencia } \\
\text { siempre que el resultado de aplicarlas se } \\
\text { aproxime al costo. }\end{array}$ & $\begin{array}{l}\text { Con respecto al sistema de valorización de los } \\
\text { costos de las existencias se puede utilizar el } \\
\text { método estándar. }\end{array}$ \\
\hline $\begin{array}{l}\text { SUBJETIVIDAD } \\
\text { VALORATIVA }\end{array}$ & $\begin{array}{l}\text { El proceso de distribución de los costos } \\
\text { indirectos fijos a los costos de conversión se } \\
\text { basará en la capacidad normal de trabajo de los } \\
\text { medios de producción. }\end{array}$ & $\begin{array}{l}\text { Con respecto a la incorporación de los costos } \\
\text { indirectos de fabricación se utiliza la tasa } \\
\text { de asignación, que requiere apreciaciones } \\
\text { subjetivas con respecto a la capacidad de planta. }\end{array}$ \\
\hline $\begin{array}{l}\text { SUBJETIVIDAD } \\
\text { VALORATIVA }\end{array}$ & $\begin{array}{l}\text { Aplicación del concepto de valor neto de } \\
\text { realización. }\end{array}$ & $\begin{array}{l}\text { Aplicación del concepto de valor neto de } \\
\text { realización. }\end{array}$ \\
\hline
\end{tabular}

Fuente: elaboración propia.

CUADRO 2. Posibilidades de contabilidad creativa en las propiedad planta y equipos

\begin{tabular}{|c|c|c|}
\hline & NICSP n. ${ }^{\circ} 17$ & NIC n. ${ }^{\circ} 16$ \\
\hline $\begin{array}{l}\text { OPCIONALIDAD } \\
\text { VALORATIVA }\end{array}$ & $\begin{array}{l}\text { Una entidad elegirá como política contable el } \\
\text { modelo del costo o el modelo de revaluación, y } \\
\text { aplicará esta política a todos los elementos que } \\
\text { compongan una clase de propiedades, planta y } \\
\text { equipo. }\end{array}$ & $\begin{array}{l}\text { Valorización posterior del activo fijo. Se } \\
\text { reconocen dos métodos para la valorización } \\
\text { del activo fijo: método costo y método al valor } \\
\text { razonable. Para el primer caso, se entiende } \\
\text { como valor libros el costo de adquisición menos } \\
\text { la depreciación, menos el importe acumulado } \\
\text { de las pérdidas por deterioro. Para el segundo } \\
\text { caso, es el valor libros el valor razonable del } \\
\text { bien menos la depreciación, menos el importe } \\
\text { acumulado de las pérdidas por deterioro. }\end{array}$ \\
\hline
\end{tabular}

\section{SUBJETIVIDAD VALORATIVA}

El costo de los elementos de propiedades, planta y equipo comprende (letra (a) en norma):

(b) todos los costos directamente relacionados con la ubicación del activo en el lugar y en las condiciones necesarias para que pueda operar de la forma prevista por la gerencia;

(c) la estimación inicial de los costos de desmantelamiento o retiro del elemento, así como la rehabilitación del lugar sobre el que se asienta, cuando constituyan obligaciones en las que incurre una entidad como consecuencia de utilizar el elemento durante un determinado periodo, con propósitos distintos del de la producción de inventarios durante tal periodo
El costo de los elementos de propiedades, planta y equipo comprende, entre otros dos conceptos (letras (a) y (b) en la norma) un tercer aspecto que dice: (c) la estimación inicial de los costos de desmantelamiento o retiro del elemento, así como la rehabilitación del lugar sobre el que se asienta, cuando constituyan obligaciones en las que incurre la entidad como consecuencia de utilizar el elemento durante un determinado periodo, con propósitos distintos del de la producción de inventarios durante tal periodo. 


\begin{tabular}{|c|c|c|}
\hline & NICSP n. ${ }^{\circ} 17$ & NIC n. ${ }^{\circ} 16$ \\
\hline $\begin{array}{l}\text { OPCIONALIDAD } \\
\text { PRESENTACIÓN } \\
\text { DE LA } \\
\text { INFORMACIÓN } \\
\text { FINANCIERA }\end{array}$ & $\begin{array}{l}\text { Cuando se revalúe un elemento de propiedades, } \\
\text { planta y equipo, la depreciación acumulada en } \\
\text { la fecha de la revaluación puede ser tratada de } \\
\text { cualesquiera de las siguientes maneras: } \\
\text { - Reexpresada proporcionalmente al cambio en } \\
\text { el importe bruto del activo en libros, de manera } \\
\text { que el importe neto del mismo en libros sea } \\
\text { igual a su importe revaluado. Este método se } \\
\text { utiliza a menudo cuando se revalúa el activo por } \\
\text { medio de la aplicación de un índice a su costo } \\
\text { de reposición depreciado. } \\
\text { - Eliminada contra el importe en libros bruto del } \\
\text { activo y se reexpresa el importe neto hasta } \\
\text { alcanzar el importe revaluado del activo. Este } \\
\text { método se utiliza habitualmente en edificios. }\end{array}$ & $\begin{array}{l}\text { Tratamiento de la depreciación acumulada en } \\
\text { caso de revalorizaciones, variará en función } \\
\text { de si se utiliza el método de eliminación de la } \\
\text { depreciación o la reexpresión de cuentas. }\end{array}$ \\
\hline $\begin{array}{l}\text { SUBJETIVIDAD } \\
\text { VALORATIVA }\end{array}$ & $\begin{array}{l}\text { El método de depreciación utilizado reflejará } \\
\text { el patrón con arreglo al cual se espera que } \\
\text { sean consumidos, por parte de la entidad, los } \\
\text { beneficios económicos futuros o potencial de } \\
\text { servicio del activo. }\end{array}$ & $\begin{array}{l}\text { Con respecto a los métodos de depreciación, la } \\
\text { norma internacional reconoce la existencia de } \\
\text { varios métodos; así el método de depreciación } \\
\text { utilizado reflejará el patrón con el cual se espera } \\
\text { que sean consumidos los beneficios económicos } \\
\text { futuros del activo. }\end{array}$ \\
\hline
\end{tabular}

Fuente: elaboración propia

CUADRO 3. Posibilidades de Contabilidad creativa en los activos intangibles.

\begin{tabular}{lll}
\hline & NICSP $\mathbf{~}^{\mathbf{0}} \mathbf{3 1}$ & NIC n. ${ }^{\mathbf{0}} \mathbf{8}$ \\
\hline $\begin{array}{l}\text { OPCIONALIDAD } \\
\text { VALORATIVA }\end{array}$ & $\begin{array}{l}\text { Capitalización de los costos de desarrollo. En la } \\
\text { norma se establece que los costos de desarrollo } \\
\text { se podrán capitalizar, estableciendo una serie de } \\
\text { requisitos asociados. }\end{array}$ & $\begin{array}{l}\text { Capitalización de los costos de desarrollo. En la } \\
\text { norma se establece que los costos de desarrollo } \\
\text { se podrán capitalizar, estableciendo una serie } \\
\text { de requisitos asociados. Cada uno de estos } \\
\text { requisitos son susceptibles de interpretarlos; por } \\
\text { ello son poco concretos los requisitos asociados a } \\
\text { la capitalización de los gastos de desarrollo. }\end{array}$ \\
\hline
\end{tabular}

Fuente: elaboración propia

Las oportunidades de contabilidad creativa en las normas internacionales de contabilidad de los sectores públicos son prácticamente las mismas que tienen las NIIF o cualquier grupo normativo de contabilidad. En ellas hay posibilidades de subjetividades y opcionalidades contables del tomador de decisiones financieras y de reporting.

\section{Conclusiones}

Con el presente estudio se pretende identificar las posibles prácticas de manipulación contable creativa al momento de aplicar las normas. Para poder determinar este aspecto, se revisó la totalidad de las Normas Internacionales de Información Financiera (NIIF) emitidas a la fecha de esta investigación, y, en efecto, estas normas permiten una variedad de posibilidades, en especial aquellas relacionadas con la regulación de partidas de activos y donde las técnicas manipuladores están mayormente centradas en la subjetividad de la aplicación de la norma por sobre la opcionalidad.

El marco conceptual de la contabilidad establece entre las características cualitativas de la información contable la comparabilidad, que se define como la capacidad de comparar los estados financieros de una empresa a lo largo del tiempo, como también de comparar los estados financieros con distintas empresas. Así, tanto la medición de los hechos económicos como la revelación de los mismos deben ser efectuadas de manera de que el analista financiero puede comparar 
distintas empresas. Ayuda a la comparabilidad la adopción de una misma gama de principios contables; atenta contra ella la existencia de la contabilidad creativa, a través de su análisis con las opcionalidades de la norma y las subjetividades de esta.

Por lo anterior, la incorporación de las Normas Internacionales de Contabilidad (NIC) y de las Normas Internacionales de Contabilidad para el Sector Público (NICSP) hacen factibles múltiples posibilidades de creatividad, orientadas a que el agente creativo refleje una posición financiera más adecuada a sus intereses, que a reflejar lo que naturalmente debe ser una organización en lo relativo a su posición financiera y a los resultados de sus operaciones.

De la misma manera que las NICSP, como cuerpo normativo, existen las subjetividades y opcionalidades, tanto de revelación como valorativas. En el caso chileno, la Contraloría General de la República de Chile, a través de la Resolución CGR n. ${ }^{\circ} 16$ de diciembre del año 2015, establece las normas de aplicación, lo cual corresponde a una adopción indirecta de las NICSP (adaptación), emitiendo una norma nacional que cumple con los requisitos de las NICSP; lo anterior, para normalizar y -en la medida que sea posible- aminorar los efectos de interpretación que llevan a subjetividades y opcionalidades de la norma.

\section{REFERENCIAS}

Amat, O. \& Blake, J. (1999). Contabilidad creativa (3a . ed.). España: Gestión 2000.

Amat, O. \& Perramon, J. (2005). Normas Internacionales de Contabilidad: NIC/NIIF (1ํ. ed.). España: Gestión 2000.

Black, E., Christensen, T., Taylor, T., \& Schmardebeck, R. (2016). The Relation between Earnings Management and Non-GAAP Reporting. Contemporary Accounting Research.

Cheng, Q., \& Warfield, T. Equity incentives and earnings management. (2005).Accounting Review, $80(2), 441-476$. Recuperado de. http://ink.library. smu.edu.sg/soa_research/829

García, M. \& Zorio, A. (2004). Posibilidad de contabilidad creativa en el marco de aplicación de las normas internacionales. Revista Quehacer Científico, 1(1), 2-21.

Griffiths, I. (1986). Creative Accounting, London: Sidfwick \& Jackson.

Healy, P., \& Wahlen, J. (1999). A review of the earnings management literature and its implications for standard setting. Accounting Horizons, 13(4), 365383.

Huber, G. (1999). Contabilidad creativa. Una aproximación empírica. Centro de Publicaciones de la Universidad Nacional del Litoral, Argentina.

Jameson, M. (1988). A practical guide to creative ac-

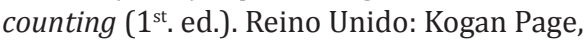

Laínez, J. \& Callao, S. (1999). Contabilidad creativa. España: Civitas.

Morales, F. \& Hollander, R. (2007). Posibilidades de aplicación de contabilidad creativa en la normativa vigente en Chile y estimación de su repercusión en los estados financieros. Panorama Socioeconómico, 34, 18-33.

Morales, F. \& Hollander, R. (2009). Contabilidad creativa en Chile. Contaduría y Administración, 229, 85-103.

Naser, K. (1993). Creative financial accounting: Its nature and use ( $1^{\text {st }}$. ed.). Reino Unido: Prentice Hall.

Pasqualini, F. \& Castel, R. (1993). Le dixième anniversaire de la loi comptable - La loi comptable, l'image fidèle et la créativité déviante. Revue de Droit Comptable, 93(1), 13-18.

Pijper, T. (1993). What is 'Creative Accounting'? In Creative Accounting. Palgrave Macmillan UK.

Rodríguez, M. C. (2001). La contabilidad creativa: análisis de la fiabilidad de la información contable. Pearson Educación.

Rodríguez-Vilariño, M. (1998). Contabilidad Creativa y factores determinantes. Técnica Contable, 50(595), 547-550.

Roychowdhury, S. (2006). Earnings management through real activities manipulation. Journal of Accounting and Economics, 42, 335-370.

Smith, T. (1992). Accounting for Growth. London: Century Business.

Stolowy, H., \& Breton, G. (2004). Accounts manipulation: A literature review and proposed conceptual framework. Review of Accounting \& Finance, 3(1), 5-66.

Vidal, M. (2002). Reflexiones en torno a la contabilidad creativa. Revista Técnica Contable, 54(647), 833845. 